\title{
XI. Auf dem Weg zur deutsch-polnischen Versöhnung
}

Neben seinem Engagement für die Belange der Kirche bot der Rahmen des Zweiten Vatikanums auch die Möglichkeit, weltliche politische Probleme einer Lösung näherzubringen. Bereits während seiner Tätigkeit für die Gesellschaft zur Förderung der inneren Kolonisation war Johannes Schauff sowohl mit den innerpolitischen Verhältnissen Polens wie auch seiner konfliktreichen Beziehung zu Preußen und dem Deutschen Reich vertraut gemacht worden; Ostsiedlung und Osthilfe waren in der deutschen innenpolitischen Auseinandersetzung auch damit gerechtfertigt worden, einen agrar- und bevölkerungspolitischen "Cordon sanitaire" gegenüber Ansprüchen und möglichen Aggressionen von seiten des östlichen Nachbarn zu schaffen. In seiner Wahlkampfzeit in Oberschlesien war Schauff zwar in erster Linie durch seine Auseinandersetzung mit dem nationalsozialistischen Gegner in Anspruch genommen worden, hatte jedoch seitdem den in der Geschichte dieser Region begründeten latenten Konfliktherd im Auge behalten.

Aufgrund seines Einsatzes auch für die polnischen "Displaced Persons“ im Rahmen seiner Tätigkeit für die ICMC hatte Schauff - dies wurde während des Konzils deutlich - das Vertrauen der polnischen Bischöfe gewinnen können. Vor allem die Beziehung zu Boleslaw Kominek - später auch Mitglied der Kurienkommission "Justitia et Pax" - gestaltete sich ausgesprochen freundschaftlich. Kominek war der Initiator und maßgebliche Autor eines Briefes der polnischen Bischöfe an ihre deutschen Amtsbrüder vom 18. November 1965, in dem angesichts des zu Ende gehenden Konzils und des bevorstehenden Millenniums der polnischen Kirche die Vergebung deutschen Unrechts offeriert und zugleich die Bitte um Vergebung eigenen Unrechts geäußert wurde ${ }^{1}$.

Um den Hintergrund auszuleuchten, vor dem dieser Brief entstand und welchen Schwierigkeiten er begegnete, ist ein knapper historischer Rückblick notwendig. Schon aufgrund der drei polnischen Teilungen 1772, 1793 und 1795 durch Rußland, Preußen und Österreich, die zum Verschwinden des polnischen Staates bis 1916 geführt hatten, war das historische Verhältnis der Polen gegenüber den Deutschen seit altersher besonders belastet. Die Einverleibung der Provinz Posen Ende 1918/Anfang 1919 sowie der die Versailler Friedensbestimmungen und internationale Abmachungen verletzende gewaltsame Versuch der Annexion Oberschlesiens Anfang der zwanziger Jahre verschärften das Verhältnis weiter. Reichskanzler Wirth (Zentrum), wenig später, wie schon angeführt, einer der politischen Mentoren Johannes Schauffs, hatte damals angesichts des polnischen Expansionsdranges und der repressiven Politik gegenüber der deutschen Minderheit gefordert, der „Versailler Bastard Polen“ müsse wieder verschwinden, und General von Seeckt, Chef der Heeresleitung, hatte erklärt, Polens Existenz sei

1 Vgl. Heller, Macht-Kirche-Politik, S. $89 \mathrm{ff}$. 
„unerträglich“ und müsse eventuell mit Hilfe Rußlands wieder beendet werden². Nach dem Machtantritt der Nationalsozialisten hatten gegenseitiges Mißtrauen und latente Aggressionsbereitschaft weiter zugenommen - trotz des Anfang 1934 abgeschlossenen Nichtangriffspaktes zwischen Polen und dem Deutschen Reich ${ }^{3}$.

Der „Hitler-Stalin-Pakt“ vom 23. August 1939 und der Kriegsausbruch anläßlich der Danzig-Frage führten zur erneuten - de facto fünften ${ }^{4}$ - Teilung Polens zwischen Deutschland und der Sowjetunion und zur Errichtung des „Generalgouvernements" mit einer brutalen deutschen Besatzungs- und Dezimierungspolitik, während die polnischen Westdistrikte mit den wichtigsten Industriezentren und 10 Millionen Einwohnern direkt dem Deutschen Reich angeschlossen wurden. Das Kriegsende, die „Westverschiebung“ Polens aufgrund des Potsdamer Abkommens der Alliierten von 1945 und die gewaltsame Vertreibung der deutschen Bevölkerung östlich der Oder-Neiße-Linie schufen die Ausgangssituation für die Entwicklung nach 1945 und bildeten zugleich erneute Imponderabilien für das künftige deutsch-polnische Verhältnis.

Die sogenannte deutsche Frage wurde in der Folgezeit zum wichtigsten machterhaltenden und politisch konsolidierenden Instrument kommunistischer Politik in Polen, auch und gerade in der Phase der Stalinisierung. Gelang es somit, auch bürgerliche politische Kräfte politisch zu pazifizieren und einzubinden, so war hier auch die national orientierte polnische Kirche beteiligt, für die die Grenzverschiebung nach Westen und die Vertreibung der Deutschen keine moralischen Probleme aufwarf. Hinzu kam die missionarische Vorstellung, die ehedem „häretischen" deutschen Gebiete könnten für den Katholizismus zurückgewonnen werden 5 .

Angesichts des tradierten, durch die Besatzungsjahre noch verstärkten „Deutschensyndroms" konnte die Gleichung wirksam werden, nach der Kommunismus = Patriotismus = Deutschenfeindlichkeit sei. Damit ließ sich der kommunistische Staat zur Inkarnation des polnischen Patriotismus stilisieren und jede Kritik an ihm als „Deutschfreundlichkeit" diffamieren. Diese Politik hatte auch negative Auswirkung auf die vor allem in Oberschlesien zurückgebliebenen Deutschen, die als "germanisierte Polen" ausgegeben und einer entsprechend rücksichtslosen Assimilationspolitik unterworfen wurden. Auf der anderen Seite stärkten die Politik der Bundesrepublik Deutschland in der strittigen Frage der „Ostgebiete" und insbesondere die Haltung der Vertriebenenverbände ihrerseits Mißtrauen und Ressentiments auf polnischer Seite. Dabei wurde nicht zur Kennt-

2 Zit. Strobel, Wirtschaftsbeziehungen, S. 111; vgl. auch Strobel, Preußenkrankheit, S. 24; Küppers, Wirth, S. $146 \mathrm{ff}$.

3 Im Zusammenhang mit polnischen Präventivkriegsdrohungen 1932/33 wurde unter Mißachtung bestehender Verträge auf der Westerplatte Danzigs, unter dem Mandat des Völkerbunds ein souveränes Völkerrechtssubjekt, eine polnische Garnison errichtet; 1934 wurde von Polen der Minderheitenschutzvertrag aufgekündigt.

4 Als vierte Teilung Polens gilt die Wiederauflösung und Aufteilung des 1807 von Napoleons Gnaden entstandenen Großherzogtums Warschau auf dem Wiener Kongreß.

5 Diese Vorstellung trug der damalige polnische Fürstprimas, Kardinal Hlond, 1946-1948 auch dem Papst vor (Strobel, Preußenkrankheit, S. 27). 
nis genommen, daß in der "Charta der deutschen Heimatvertriebenen" schon 1950 jeglicher Verzicht auf Gewalt erklärt worden war.

Die Ost- und Polenpolitik Adenauers war zum einen durch innenpolitische Rücksichtnahmen auf die Heimatvertriebenen bestimmt, zum andern jedoch, wie Hans-Peter Schwarz formuliert, „von Grund auf revisionistisch“6. Ein von Johannes Schauff und Kardinal Frings überliefertes unerfülltes Vermächtnis Adenauers, nach dem neben der Versöhnung mit den Juden und den Franzosen vor allem der Ausgleich mit den östlichen Nachbarn, allen voran den Polen, herbeigeführt werden sollte ${ }^{7}$, scheint in Hinblick auf Polen doch eher als Fiktion. Die Beziehungen zu Polen waren von vornherein belastet durch die Frage der deutschen Einheit bzw. der Existenz der DDR. Schließlich wurde mit der Formulierung der „Hallstein-Doktrin“ in der Regierungserklärung vom 28. Juni $1956^{8}$ die Polenpolitik wie die gesamte Osteuropapolitik zu einer Funktion der Deutschlandpolitik. Diese zu bewegen, war nur mit Moskau möglich?.

An der Nichtanerkennung der Oder-Neiße-Grenze hielt die Bundesregierung bis Anfang der siebziger Jahre und am Friedensvertragsvorbehalt bis 1990 fest. Bemühungen um eine flexiblere Haltung - etwa von Außenminister Brentano Mitte der fünfziger Jahre ${ }^{10}$ - standen nach wie vor im Kontext einer Zurückdrängung des Kommunismus. Gerade weil der Katholik Heinrich von Brentano aufgrund der nationalsozialistischen Verbrechen gegenüber Polen eine besondere moralische Verpflichtung für Deutschland sah ${ }^{11}$, schien es nicht tolerabel, daß die Polen nun erneut unter einem totalitären Regime leben mußten. Einer möglichen deutsch-polnischen Annäherung maß Brentano deshalb großes Gewicht bei, weil sich Polen damit möglicherweise „aus der Umklammerung zwischen der Sowjetunion und der Sowjetzone zu befreien" vermochte ${ }^{12}$.

Auch wenn der junge CDU-Politiker Ernst Majonica schon 1951 in Paris (exil-)polnische und deutsche Christdemokraten zusammengebracht hatte und der SPD-Bundestagsabgeordnete Carlo Schmid 1958 einen Gastvortrag an der Warschauer Universität hatte halten können und bei dieser Gelegenheit auch mit einem Vertreter der polnischen Kirche zusammengetroffen war ${ }^{13}$, erfolgte in der westdeutschen Öffentlichkeit erst ab Beginn bzw. Mitte der sechziger Jahre eine Öffnung gegenüber den östlichen, insbesondere dem polnischen Nachbarn. Sie ging von akademischen, publizistischen und kirchlichen Kreisen aus und war ein

6 Schwarz, Konrad Adenauer. Der Staatsmann, S. 945.

7 Diese politischen Zielvorgaben hatte Adenauer offenbar ursprünglich gegenüber Schauff geäußert (Schauff, Autobiographische Notizen/Fragmente). Frings hatte von diesem Vermächtnis Adenauers anläßlich eines Vortrages am 28. 4. 1970 im Wilhelm-Böhler-Klub gesprochen (ein entsprechender Vermerk von Herbert J. Becher/Katholisches Büro, Anlage zu einem Brief Bechers an Schauff vom 22. 5. 1970), IfZ, NL Schauff, Bd. 26.

8 Außenpolitik der Bundesrepublik Deutschland, S. $231 \mathrm{f}$.

9 Vgl. Schwarz, Das außenpolitische Konzept Adenauers, in: Morsey/Repgen (Hrsg.), Adenauer-Studien, Bd. I, S. 92.

$10 \mathrm{Vgl}$. Bingen, Polenpolitik, S. $42 \mathrm{f}$.

11 Vgl. Kosthorst, Brentano, S. 167.

12 Ebenda, S. 46.

13 Die Reise hatte heftige Vorwürfe von Parteifreunden und Vertriebenenvertretern provoziert, vgl. Schmid, Erinnerungen, S. 628. 
vorerst individuelles und gewissermaßen „von unten“ ausgehendes Engagement ${ }^{14}$. Auch Politiker wie Herbert Czaja sind hier zu nennen, den seine Funktion als Vertriebenenpolitiker nicht daran hinderte, den Polen helfen zu wollen allerdings im Sinne einer Überwindung der kommunistischen Diktatur.

Auch Johannes Schauff hatte sich bereits frühzeitig in dieser Richtung bemüht. Von polnischer Seite sind Politiker wie Stanislaw Stomma, Repräsentant der katholischen Gruppe ZNAK im Sejm, und Jerzy Turowicz, Chefredakteur der katholischen Wochenzeitung "Tygodnik Powszechny“, zu nennen. Stomma war bereits 1958 als stellvertretender Vorsitzender des Auswärtigen Ausschusses des Sejm in Deutschland mit Außenminister von Brentano zusammengetroffen. Auch mit Czaja, Guttenberg und Majonica hatte er Gespräche geführt ${ }^{15}$.

In dem Gespräch zwischen Brentano und Stomma, über das auch Johannes Schauff informiert wurde, erkannte der Außenminister de facto die neuen Grenzen an, obwohl er dies "nicht offiziell“ sagen könne ${ }^{16}$. Die Schwierigkeit, aufeinander zuzugehen, bestand darin, daß die deutsche Seite nicht akzeptieren wollte, daß die polnische Kirche trotz ihrer Konflikte mit der kommunistischen Regierung mit ihr in der Grenzfrage einig war, während die Polen hinter der innenpolitisch motivierten Bonner Tendenz, die Grenzfrage offenzuhalten, eine präzise außenpolitische Strategie vermuteten ${ }^{17}$.

$\mathrm{Zu}$ einer weiteren allmählichen Annäherung kam es auf der Ebene der polnischen und der deutschen katholischen Kirche - auch wenn Kardinalprimas Wyszynski anläßlich der Feiern zum 20. Jahrestag der Übernahme der ostdeutschen Gebiete (31. August bis 2. September 1968) noch einmal vehement ihre historische Zugehörigkeit zu Polen beschwor und ihre „Rückkehr zu Polen“ als eine „sichtbare Intervention Gottes“ bewertete ${ }^{18}$.

Weniger exponiert und aus eigener Erfahrung zu einer differenzierten Sicht der Deutschen befähigt war der damalige Titularerzbischof in Breslau, Boleslaw Kominek. Dem 1903 in Schlesien geborenen und aufgewachsenen Kominek war das Mißtrauen gegen jegliche Art von Nationalismen buchstäblich eingebleut worden - in der deutschen Schule, wenn er polnisch, und im polnischen Elternhaus, wenn er deutsch sprach $^{19}$.

Kominek war schon früher ein offener Ansprechpartner für die Deutschen gewesen, die Ende der fünfziger/Anfang der sechziger Jahre etwas zahlreicher Polen und auch die Westgebiete besucht hatten. Darunter waren u. a. auch die protestantischen Pastoren Niemöller und Albertz. Der bis dahin einzige Kontakt des polnischen Episkopats zu einem deutschen Amtsbruder war der zu dem Danziger Bischof Carl Maria Splett gewesen, der bis 1956 vom kommunistischen Regime

\footnotetext{
14 Vgl. Bingen, Polenpolitik, S. $87 \mathrm{ff}$.

15 Vg. Pailer, Stanislaw Stomma, S. 77; Bingen, Polenpolitik, S. $59 \mathrm{f}$.

16 „Stanislaw Stomma, Zum Thema deutsch-polnische Normalisierung“, Interview mit St. Stomma, in: Informationsdienst des katholischen Arbeitskreises für zeitgeschichtliche Fragen, Nr. 140 (1986), S. 77-83. Das Gespräch wurde von Schauff gegenüber Edith Heller bestätigt: Heller, Macht-Kirche-Politik, S. 80.

17 Vgl. Stehle, Seit 1960, S. 155.

18 Zit. nach Heller, Macht-Kirche-Politik, S. 70 ff.; Strobel, Preußenkrankheit, S. 29.

19 Stehle, Seit 1960, S. 158.
} 
inhaftiert war und sich bei den polnischen Bischöfen für seine Freilassung bedankt hatte. Zum Fest der Heiligen Hedwig am 16. Oktober 1960 hatte sich der damalige Bischof von Berlin, Julius Döpfner, um ein erstes deutliches Zeichen der Versöhnung angesichts des Leides bemüht, das beide Völker hatten erdulden müssen. Nach allem, was im Namen des deutschen Volkes geschehen sei, könne der Frieden nur unter großen Opfern erlangt werden. Dabei sei die Zukunft der Gemeinschaft der Völker und Staaten wichtiger als Grenzfragen ${ }^{20}$. Die Predigt Julius Döpfners in der St. Eduard-Kirche empfand Jerzy Turowicz als „zutiefst christliche Stimme", wenn auch der Vergleich von deutscher und polnischer Schuld zurückgewiesen wurde. Die polnische Nation sei bereit, Döpfners Angebot zur Versöhnung zu folgen ${ }^{21}$.

Nicht zuletzt das Zweite Vatikanische Konzil ermöglichte es den polnischen Bischöfen, ihre deutschen Amtsbrüder auch persönlich kennenzulernen. Eine der wichtigsten Begegnungsstätten in diesem Zusammenhang bildete die Schauffsche Wohnung in der Via Gregoriana.

Kominek war, wie schon angeführt, Initiator und maßgeblicher Autor des Briefes der polnischen Bischöfe an ihre deutschen Amtsbrüder vom 18. November 1965, der in der Tat eine ausgestreckte Hand, einen Versöhnungsbrief also darstellte. Zugleich war er ein frommer, wenn auch diplomatisch vielleicht nicht ganz geschickter Versuch, „mit religiösen, moralischen und historischen Argumenten, aber auch mit psychologischem Verständnis für die vertriebenen Ostdeutschen, die Oder-Neiße-Grenze akzeptabel zu machen"22.

Bei dieser Versöhnungsinitiative, der der polnische Kardinalprimas Wyszynski mit skeptischer Distanz gegenüberstand, wurde Kominek nach eigener Aussage auch durch seine Gespräche mit Schauff ermutigt ${ }^{23}$. Die Antwort der deutschen Bischöfe, die am 5. Dezember 1965 erfolgte, stimmte zwar auch in den Tenor von Frieden und Versöhnung ein, blieb aber in der Frage der Oder-Neiße-Grenze unbeweglich. Die deutschen Bischöfe fanden sich zur Enttäuschung Komineks nicht bereit, das Heimatrecht von acht Millionen Polen in den Westgebieten anzuerkennen.

Der Brief, so Edith Heller, steckte - sicherlich ungewollt und unbewußt - voller Ressentiments ${ }^{24}$. Dies erstaunt um so mehr, als Döpfner bereits 1960 eine viel klarere Sprache gesprochen hatte. Nunmehr im Amt des Vorsitzenden der Deutschen Bischofskonferenz, schienen wieder alte Komplexe des Katholizismus aus der Kulturkampfzeit zum Tragen zu kommen - man wollte nicht als national unzuverlässig gelten ${ }^{25}$. Schauff hatte Döpfner verschiedentlich getroffen, auch in Rom,

20 Zit. nach: Versöhnung, S. 25 f.

21 Zit. nach Heller, Macht-Kirche-Politik, S. 82.

22 Hansjakob Stehle, Der Klerus und die deutsch-polnische Aussöhnung. Eine bittere Epistel, in: Die Zeit, Nr. 52, 22. 12. 1978.

23 Hansjakob Stehle, Johannes Schauff, S. 248. Auch der damals in Rom weilende Publizist Walter Dirks war von Kominek konsultiert worden, der die Initiative des Bischofs ebenfalls unterstützte (Heller, Macht-Kirche-Politik, S. 91 f.).

24 Heller, Macht-Kirche-Politik, S. 120.

25 Vgl. Schreiben Kardinal Döpfner an Kardinal Wyszynski vom 15.5. 1971 (IfZ, NL Schauff, Bd. 26). 
und den Kardinal hin und wieder über die Resonanz der polnischen Initiativen im Kreis seiner politischen Freunde ins Bild gesetzt ${ }^{26}$. Männer wie der Kirchenhistoriker Hubert Jedin, der vor einem „nationalistisch verzerrten Geschichtsbild“ der Polen warnte, hatten hier aber offenbar größeren Einfluß27.

Der polnische Episkopat geriet indessen aufgrund seiner Versöhnungsinitiative und der unbefriedigenden Antwort von deutscher Seite ins Feuer kommunistischer Propaganda, die ihn wegen "nationaler Unzuverlässigkeit“ denunzierte. Vorgänge wie die Ablehnung Döpfners, mit Kominek, der über die Vorgänge in seiner Heimat zutiefst erschrocken war, Anfang Januar 1966 zusammen im Deutschen Fernsehen aufzutreten, um dem bedrängten polnischen Episkopat beizustehen, mußten diese Situation noch weiter verschärfen ${ }^{28}$.

Durch diese Entwicklung nicht wenig enttäuscht, nutzte Schauff seine Verbindungen zunächst zur Organisation praktisch-humanitärer Hilfe, vor allem auch für polnische NS-Opfer ${ }^{29}$. Mit Unterstützung Komineks war er bemüht, die deutschsprachige Seelsorge in den polnischen Westgebieten zu verbessern, so wie es auch eines seiner Anliegen war, die deutschsprachige seelsorgerische Betreuung der Rückwanderer im Südtiroler Fersental zu sichern ${ }^{30}$. Beide Bemühungen entsprachen der Überzeugung Schauffs, daß „Siedlung“ und „christliches Bauerntum“ als Fundament zur Integration, aber auch für die Bewahrung kultureller und religiöser Identität eine maßgebliche nationale und ideelle Funktion besäßen. Daß dies auch dem Schutz bedrohter nationaler bzw. ethnischer Identitäten dienen könne, war zentrales Thema Schauffs in seinen Vorschlägen zur „Realisierung der Menschenrechte" in Polen und den übrigen Ostblockstaaten, die er auch als Mittel zum Zweck der Verbesserung der Lage der deutschen Minderheiten sehen wollte ${ }^{31}$.

Eine für die beiderseitige Verständigung nützliche Unternehmung war das am 26. März 1969 in erster Linie mit Blick auf Polen begründete „Werk für Europäische Partnerschaft". Das Projekt war bereits im Sommer 1968 in Rom initiiert worden und ging wesentlich auf Schauffs Initiative zurück, der am 29. Oktober 1968 im Berliner Reichstag das entscheidende Gespräch zu dessen Unterstützung von deutscher Seite zwischen Czaja, Wehner und Rechtsanwalt Frey vom Katholischen Büro arrangiert hatte. Das Projekt stand im Rahmen der von Schauff bereits während seiner Tätigkeit für die „Fédération Internationale de Solidarité“ begonnenen geistigen Offensive gegenüber dem kommunistischen Osten ${ }^{32}$. Bei

26 Korrespondenz Schauff-Kardinal Döpfner, 1965 (IfZ, NL Schauff, Bd. 26).

27 Jedin, Lebensbericht, S. 216; Heller, Macht-Kirche-Politik, S. 112.

28 Am 5.1. 1966 gab Kominek statt dessen Stehle ein Fernsehinterview, das am 10.1. von Joachim Fest in der Sendung „Panorama“ präsentiert wurde. Darin wird von ihm entschieden verneint, daß die Oder-Neiße-Grenze das Objekt von Gesprächen und Verhandlungen sein könne; der Potsdamer Beschluß sei für Polen ein ganz positiver Friedensschluß (zit. nach Stehle, „Versuchen wir zu vergessen“, in: Feinde werden Freunde, S. 82 f.; vgl. auch Heller, Macht-Kirche-Politik, S. 165).

29 Siehe unten, S. $184 \mathrm{ff}$.

30 Hierüber ein Briefwechsel mit Herbert J. Becher mit der Anlage des Vortrags des Problems durch Peter Pompermeier vom 20. 2. 1975 und dem Schreiben Schauffs an Josef Prader vom 4. 3. 1975 (IfZ, NL Schauff, Bd. 1).

31 Vgl. Schauffs Aide-mémoire „Ostpolitische Schritte“, o. D., IfZ, NL Schauff, Bd. 40.

32 Siehe, S. $141 \mathrm{ff}$. 
seiner Realisierung waren von Anfang an Herbert Czaja und Vertreter des Katholischen Büros des Kommissariats der deutschen Bischöfe beteiligt - dieselben Personen im übrigen, die auch bei Schauffs Bemühungen zur Lösung des Südtirol-Problems beteiligt waren.

Das „Werk für Europäische Partnerschaft“ wurde erst durch das nach Bildung der Großen Koalition veränderte politische Klima denkbar, und die in die Polenpolitik der Bundesrepublik involvierten deutschen Politiker - Brentano, Guttenberg, Wehner - waren enge Vertraute von Johannes Schauff. Von deutscher Seite wurden weiterhin die „Pax-Christi“-Bewegung (Reinhold Lehmann), die katholischen und protestantischen Kirchenvertreter Tenhumberg und Dibelius sowie der Kölner Generalvikar Josef Teusch aktiv. Die polnischen Partner waren Erzbischof - später Kardinal - Kominek und die Bischöfe Wesoly und Rubin; dazu kam als Vertreter des Deutschen Caritasverbandes Josef Pawliczek.

Nahziel war neben einer publizistischen Aufklärungsoffensive vor allem ein akademisches Stipendienprogramm für polnische Studenten, das der damalige Krakauer Erzbischof und nachmalige Papst Johannes Paul II., Karol Wojtyla, in einem Gespräch mit Johannes Schauff am 9. Oktober 1968 angeregt hatte: Wojtyla hatte sich über die Ausgrenzung polnischer Studenten an westlichen Universitäten beklagt, die nicht als politische Flüchtlinge gelten wollten; angesichts des furchtbaren Aderlasses der geistigen polnischen Eliten durch den nationalsozialistischen wie den stalinistischen Terror sowie angesichts der nach wie vor wirksamen kommunistischen Repression sei eine solche Ausbildung von künftigen Priestern und Laien im Westen für das polnische Episkopat von erstrangiger Bedeutung. Empfänger sowie Herkunft der Stipendien sollten wegen möglicher politischer Mißdeutungen vertraulich behandelt werden ${ }^{33}$.

Die Unternehmungen des „Werks für Europäische Partnerschaft“ wurden vom Auswärtigen Amt finanziert: Bis 1990, dem Todesjahr von Johannes Schauff, wurden etwa zwei Millionen DM vor allem in Projekte individueller Förderung investiert $^{34}$. Vom Außenministerium war Dr. Arnold und vom Bundesvertriebenenministerium Dr. Nahm mit den Vorgängen befaßt ${ }^{35}$. Organisatoren vor Ort waren in Paris Maria Winowska, eine Vertraute Wyszynskis und Mitarbeiterin von Bischof Rubin, die für die Studenten im Ausland zuständig war ${ }^{36}$; Pater Paulus Gordan oblag die Organisation eines Bücherfonds.

33 Insgesamt sollten 16000 DM für solche Stipendien zur Verfügung gestellt werden (Vermerk über ein Gespräch mit Kardinal Woytila, Erzbischof von Krakau, IfZ, NL Schauff, Bd. 26). Bei der in der Niederschrift erwähnten "Vertrauensperson des polnischen Episkopats, die in Westeuropa (Paris) tätig ist “ und die ebenfalls an dem vertraulichen Gespräch teilnahm, handelt es sich um Maria Winowska, die für das Stipendienprogramm verantwortlich war.

34 Schreiben von Becher an Michael Schauff vom 23. 5. 1990 (IfZ, NL Schauff, Bd. 32).

$35 \mathrm{Vgl}$. Schreiben Schauff an Guttenberg, damals Staatssekretär im Bundeskanzleramt, vom 15. 1.1968 betr. Schauffs Zusammenarbeit mit Nahm und seine Kontakte zu dem Breslauer Bischof Kominek u.a. in der Frage der Diözesan-Neugliederung in den polnischen Westgebieten (BA, NL Guttenberg, Bd. 42, Bl. 103).

36 Briefwechsel Maria Winowska-Familie Schauff 1970-1979 (IfZ, NL Schauff, Bd. 26). 
Am 10. November 1968 wurde Schauff zusammen mit Pater Paulus Gordan von dem polnischen Kardinalprimas Wyszynski empfangen. Schauff hatte Wyszynski bereits früher kennengelernt. Anläßlich einer solchen Begegnung hatten beide einen gemeinsamen guten Bekannten festgestellt: Max Josef Metzger, Mitbegründer des Friedensbundes deutscher Katholiken bzw. der „Pax-Christi“Bewegung und engagierter Ökumeniker, der wegen seines pazifistischen Engagements nach 1933 mehrfach verhaftet und am 17. April 1944 von den Nationalsozialisten hingerichtet worden war. Schauff hatte Metzger schon 1923 auf dem von Marc Sangnier organisierten Friedenskongreß in Freiburg im Breisgau kennengelernt ${ }^{37}$. Wyszynskis Freundschaft mit Metzger stammte von einem längeren Aufenthalt in Metzgers Heimat Meitingen, wo jener ihm deutschen Sprachunterricht erteilt hatte. Angesichts dieses gemeinsamen Hintergrunds habe ihn Wyszynski, wie Schauff berichtet, spontan umarmt und sei vom Italienischen ins Deutsche gewechselt; seitdem habe ein enges persönliches Verhältnis bestanden, das es Schauff auch erlaubt habe, zugunsten des von Kominek entworfenen Versöhnungsbriefs zu intervenieren ${ }^{38}$.

Bei dem vertraulichen Treffen am 10. November, das etwa 40 Minuten dauerte, war allerdings das Stipendienprogramm, das der Kardinal sehr positiv bewertete, nur der Ausgangspunkt für eine politische Tour d'horizon im Brennpunkt des Jahres 1968. Es war dies das Jahr der westlichen und polnischen Studentenunruhen, des „Prager Frühlings" und seines (auch mit polnischer Beteiligung) herbeigeführten schnellen Endes. Im Unterschied zu den Pariser Mai-Ereignissen, so Wyszynski zu Schauff, müsse man die polnischen Studentenproteste „als den disziplinierten Ausdruck des Wunsches nach Studienreform und größeren Freiheiten innerbalb der bestehenden Ordnung auffassen“. Die kommunistische Ideologie besitze in Polen keine überzeugten Anhänger mehr, der Kommunismus habe als politisch-wirtschaftliches System vollständig versagt und sich statt dessen in eine "sterile Verwaltungsbürokratie" verwandelt.

Wyszynski beklagte die generelle Unfreiheit aller Ostblockstaaten und die drückende Präsenz sowjetischer Truppen, worauf Schauff auf die "Gefahr eines Illusionismus" im Umgang mit dem Osten hinwies. Bei seiner Tätigkeit für die

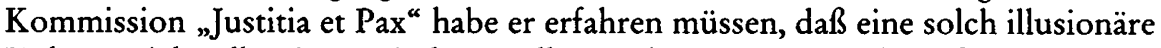
Haltung sich selbst in römischen Stellen ausbreite. Wyszynski äußerte sich unbefriedigt über den Stand der polnisch-deutschen Beziehungen, vor allem über die nach wie vor ausstehende kirchenrechtliche Regelung in den polnischen Westgebieten. Dies gebe dem Regime, das überhaupt keine sachliche Lösung anstrebe, sondern immer nach einem Anlaß suche, die "deutsche Gefahr" anzuprangern, die Handhabe, zugleich die Kirche zu verdächtigen. Schauff bat seinerseits den Kardinal um Verständnis dafür, daß dieser hochpolitische Fragenkomplex mit aller Umsicht angegangen werden müsse und „daß eventuelle Konzessionen, zu denen sich die Bundesregierung am Rande des Konkordats Polen gegenüber in Sachen der westpolnischen kirchlichen Jurisdiktionsgebiete bereit erklären

37 Siehe, S. 36.

38 „Dr. Max Josef Metzger und Kardinal Wyszynski“. Memorandum Schauff vom 17.7. 1987 (IfZ, NL Schauff, Bd. 26). 
würde, [nicht] etwa automatisch der DDR zugute kämen. Dafür erbat und erhielt er das volle Verständnis des Kardinals." 39

Die Jahre der Großen Koalition 1966 bis 1969 bildeten in der Ost- und Polenpolitik eine Zeit des Übergangs, in der eine Revision der „alten“ Ostpolitik nur halbherzig betrieben wurde ${ }^{40}$. Kanzler Kiesinger selbst suchte den politischen Ausgleich mit Polen allenfalls auf der Grundlage des Status quo, während die Sozialdemokraten weiterhin völkerrechtliche Vorbehalte gegen eine Grenzanerkennung formulierten. Doch wurde von ihnen zugleich die vorläufige Unantastbarkeit der bestehenden Grenze herausgestellt ${ }^{41}$. Brandt sprach der Aussöhnung mit Polen den gleichen Rang zu wie der mit Frankreich - eine Haltung, die nach dem Wechsel zur sozial-liberalen Koalition den Weg zum Warschauer Vertrag ebnete.

Bevor es jedoch am 7. Dezember 1970 zur Unterzeichnung dieses Vertrages kam, der die "Oder-Neiße-Linie“ als westliche Grenze der Volksrepublik Polen anerkannte und die „Unverletzlichkeit“ der „bestehenden Grenzen“ der beiden Staaten "jetzt und in aller Zukunft" bekräftigte"2, war am 12. August 1970 der deutsch-sowjetische Vertrag unterzeichnet worden. Die Verhandlungen waren zeitlich parallel gelaufen, und dies hatte bei den Polen im Vorfeld der Verhandlungen mit Deutschland Ängste geschürt, wie 30 Jahre zuvor Opfer deutsch-sowjetischer Verträge zu werden. Besorgte Fragen des polnischen Episkopats, welche Gespräche und Verhandlungen hierbei denn „Vorrang“ besäßen, gab Schauff nach Bonn weiter. Am 14. Juli 1970, einen Tag nach dem Besuch von Kanzler Brandt im Vatikan und vier Wochen vor Unterzeichnung des Moskauer Vertrags, wurde Kardinal Wyszynski ein ganz offensichtlich von Schauff selbst abgefaßtes „Aidemémoire“ zugeleitet - „zur Kenntnis auch von Kardinal Wojtyla und Erzbischof Kominek“ -, in dem es heißt: „Die deutsche Regierung hatte die Absicht, den Vertrag mit Polen direkt und vor dem Vertrag mit Moskau zu schließen ... um jeder möglichen Fehlinterpretation vorzubeugen, nach der Deutschland und Rußland wie schon so häufig in der Vergangenheit - über das Schicksal Polens bestimmen wollten. Aber Moskau hat bei der polnischen Regierung darauf bestanden, den Vertrag mit Bonn erst nach einem solchen zwischen Moskau und Bonn zu schließen. Herr Gomulka mußte dies akzeptieren. Die deutsche Seite bemüht sich trotzdem und in der Hoffnung auf Erfolg, die beiden Verträge zu synchronisieren ..."43

39 Gedächtnisprotokoll über ein Gespräch zwischen Kardinal Wyszynski, Schauff und P. Paulus Gordan im Polnischen Kolleg, Rom, 10.11. 1968 (IfZ, NL Schauff, Bd. 26).

40 Vgl. Bingen, Polenpolitik, S. 99.

41 Diese Vorbehalte betonte besonders Wehner. Vgl. Texte zur Deutschlandpolitik, Bd. 1, S. 114; Bingen, Polenpolitik, S. 101.

42 Zum Vertrag, seiner Rezeption und zur Ratifizierungsdebatte in Deutschland und im Bundestag vgl. Bingen, Polenpolitik, S. $134 \mathrm{ff}$.

43 Das Dokument ist in Französisch abgefaßt (Übersetzung durch den Verfasser), sein Autor ist die „deutsche Person, die in Rom ständig mit Ihnen (Wyszynski) und anderen Mitgliedern des polnischen Episkopats in Kontakt stand, nach einem Gepräch mit Herrn Brandt anläßlich seines Besuchs im Vatikan“ (IfZ, NL Schauff, Bd. 26). 
Die polnischen Bischöfe hatten darauf gehofft, daß die von der sozialliberalen Regierung aufgenommenen deutsch-polnischen Verhandlungen auch zu der überfälligen kirchenrechtlichen Neuordnung in den Oder-Neiße-Gebieten führen würden. Dieses Thema war schon anläßlich der Besuche der SPD-Politiker Wehner und Leber 1968 im Vatikan erörtert worden, und Kominek stand seither durch die Vermittlung von Schauff mit beiden Politikern inoffiziell in Kontakt; er besaß dazu das ausdrückliche Plazet von Wyszynski.

Ende November 1969 hatten zwei Treffen in Schauffs römischer Wohnung in der Via Gregoriana stattgefunden. Nun zerstob allerdings auch die von Papst Paul VI. in Bonn angeregte Möglichkeit, wenigstens die Regelung der polnischen Diözesanfrage vertraglich vorzuziehen - Bundeskanzler Brandt riet aus innenpolitischen Rücksichten zum Abwarten. „Die Frage der Diözesen in den Westgebieten“ - so heißt es weiter in dem Aide-mémoire - „war nicht auf der Tagesordnung der Begegnung zwischen Herrn Brandt und dem Heiligen Vater; denn nach dem Besuch der Herren Wehner und Leber und ihren Vorstößen ist das Problem durch eine böswillige Agitation von Seiten der CDU, der Flüchtlingsverbände und selbst einiger Bischöfe verschärft worden - so als wollte sich die sozialliberale Regierung für ihre Ostpolitik zum Schaden der deutschen Interessen des Heiligen Stuhls bedienen."

Als sich die deutschen Christdemokraten am 16. Oktober 1970 endlich für eine "neue Polenpolitik“ aussprachen, wurde ihnen am 28. Oktober 1970 über Schauff ein Katalog Komineks mit zehn kritischen Fragen und Feststellungen übermittelt. Darin werden schwere Vorwürfe gegenüber der CDU/CSU-Führung erhoben, die in ihrer sechzehnjährigen Regierungszeit keine Initiativen gegenüber Polen ergriffen und bestehende Chancen nicht genutzt habe: „Warum hat man die angebotene Vermittlung von de Gaulle nicht angenommen?" - ja, sich nicht einmal um Kontakte bemüht: „Jetzt hat die deutsche Linke unter Brandt die längst fällige Frage in die Hand genommen. Soll deshalb das historisch-ethisch Notwendige und Zukunftsträchtige unterlassen werden? ... Warum haben die katholischen Politiker der CDU/CSU besonders nach dem Briefwechsel der Episkopate beider Länder während des Konzils keine eigenen Initiativen entwickelt? Warum haben sie sogar am stärksten die Konsolidierung der Seelsorge der katholischen Kirche in den Westgebieten bekämpft, unter Berufung auf das ,Hitlerkonkordat'? Warum hat die evangelische Kirche viel positiver auf die Botschaft des Episkopats während des Konzils reagiert als die deutschen Bischöfe? Warum gibt es keine ähnlich mutigen Äußerungen wie die von Richard von Weizsäcker von katholischen Politikern der CDU/CSU? Wie soll da der ,Wille zum Dialog' glaubhaft werden?" 44

Dies waren Fragen, die Schauff nicht nur übermittelte, sondern die ihn auch selbst bewegten. Vor allem die von Kominek erwähnten früheren Bemühungen von Außenminister Brentano im Jahre 1957, die von Minister Oberländer und den Vertriebenenverbänden konterkariert worden waren, hatte er aufgrund seines

44 „Fragen und Einwände gegen die von der CDU/CSU-Fraktion am 16. 10. 1970 beschlossene ,Neue Polenpolitik“" (Übers. aus dem Polnischen). Das Dokument wurde auch anderen christdemokratischen Parteien verschiedener europäischer Staaten übermittelt (IfZ, NL Schauff, Bd. 26); vgl. auch Heller, Macht-Kirche-Politik, S. 175 f. 
engen Kontaktes zu Brentano noch in lebhafter Erinnerung. So kam es - nach Vertragsabschluß zwischen Bonn und Moskau am 12. August 1970 und noch vor Beginn der deutsch-polnischen Verhandlungen am 2. November - wesentlich auf Initiative Schauffs am 14. Oktober 1970 in Rom zu einem vertraulichen Treffen zwischen Döpfner und Wyszynski, der von Kominek und Kardinal Wojtyla begleitet wurde.

Die polnischen Kirchenführer standen vor dem Hintergrund drohender nationaler Diskreditierung durch das kommunistische Regime in der Diözesanfrage unter Erfolgszwang. Das für die Polen enttäuschende Ergebnis dieser Zusammenkunft dokumentiert ein Brief Wyszynskis an Kardinal Döpfner vom 5. November 1970 , den Wyszynski Johannes Schauff in Kopie überließ ${ }^{45}$. Wyszynski, der auch den Papst nicht für eine Vorentscheidung hatte gewinnen können ${ }^{46}$, äußerte in seinem Schreiben die Enttäuschung, welche die Anwort der deutschen Bischöfe auf den polnischen Versöhnungsbrief ausgelöst habe und wies auf das viel positivere Entgegenkommen der deutschen Protestanten hin. Dabei sei die polnische Kirche ein Bollwerk des Katholizismus im Osten und die Grenzfrage sowohl eine Kirchen- als auch Lebensfrage. Auch daß der Heilige Stuhl sich nicht in der Lage sehe, die Diözesanfrage in den Westgebieten zu lösen, lag für Wyszynski im Widerstand des deutschen Episkopats begründet. Solange diese Frage ungelöst bleibe, gebe „es keinen Fortschritt auf dem Weg der Versöhnung und friedlichen Zusammenarbeit".

Der ob des Tenors dieses Schreibens erschreckte Döpfner antwortete am 14. Dezember, eine Woche nach Unterzeichnung des Warschauer Vertrags ${ }^{47}$. Das Antwortschreiben zeigte zwar Betroffenheit ob der harschen Kritik des polnischen Kardinalprimas, in der Frage der westlichen Diözesangrenzen wollte Döpfner jedoch an dem „bewährten Brauch“ der Kirche festhalten, erst politische Lösungen abzuwarten. Auch sah er weiterhin den gemeinsamen historischen Schuldkomplex von nationalsozialistischen Verbrechen in Polen und der Vertreibung der Deutschen. Nachdem mit dem Warschauer Vertrag bzw. mit dessen Ratifizierung die politischen Voraussetzungen gegeben waren, zögerte Döpfner nicht mehr, der kirchlichen Neuregelung in den polnischen Westgebieten durch den Heiligen Stuhl und in Zusammenwirkung mit den zuständigen politischen Instanzen auch von der Seite des deutschen Episkopats grünes Licht zu geben ${ }^{48}$. Am 28. Juni 1972 dekretierte der Vatikan die Neuordnung der Kirchenverwaltung in den West- und Nordgebieten und die Anpassung der Diözesangrenzen an die Oder-Neiße-Grenze ${ }^{49}$.

Gleichwohl bestand bei den deutschen Bischöfen ein grundsätzlicher innerer Vorbehalt weiter, da eine definitive friedensvertragliche Regelung nach wie vor

45 IfZ, NL Schauff, Bd. 26.

$46 \mathrm{Vgl}$. Stehle, Wyszynski und Döpfner, S. 544.

47 Der Brief Döpfners in IfZ, NL Schauff, Bd. 26.

$48 \mathrm{Vgl}$. Döpfners Rede vor einer ZNAK-Delegation in der Katholischen Akademie in Bayern am 13. 6. 1972 (zit. nach Stehle, Vatikan, S. 559-566).

49 Die Neueinteilung bei Heller, Macht-Kirche-Politik, S. 184; Heller weist darauf hin, daß die östlichen Diözesangrenzen mit der Staatsgrenze nach wie vor nicht übereinstimmen. 
ausstand. Auch von polnischer Seite gab es ein weiterhin anhaltendes Mißtrauen, das durch nach dem Vertrag erleichterte gegenseitige Besuche nur langsam abgebaut wurde. Hier versuchte Johannes Schauff wiederum mit Aktionen zu helfen, die sowohl seine Großherzigkeit als auch seine Hilflosigkeit angesichts dieses Mißtrauens vor dem Hintergrund der beiderseitigen historischen Verletztheiten dokumentieren. Über Prälat Kaas war das Brustkreuz von Papst Gregor XVI.50, das diesem von polnischen Katholiken geschenkt worden war, in den Besitz der Familie Schauff übergegangen. Nach Beratung mit Monsignore Benelli wollte Schauff dieses kostbare Insignium an die Polen zurückgeben.

„Meine Frau und ich“, berichtete er am 14. November 1973 an Benelli, „haben das Brustkreuz Kardinal Boleslaw Kominek, Erzbischof von Wroclaw (Breslau), anläßlich der Übernahme seiner Titelkirche am 21. Oktober 1973 in Rom übergeben. Dies soll eine symbolische Geste persönlicher deutsch-polnischer Wiedergutmachung sein. Als Mitglied des Deutschen Reichstags der katholischen Zentrumspartei vor 1933 für den Wahlkreis Liegnitz in Schlesien schien mir die Rückgabe an den neuen Kardinal und Erzbischof von Wroclaw, zu dessen Diözese Liegnitz auch heute noch gehört, besonders angebracht und sinnvoll zu sein. In diesem Zusammenhang sehe ich auch meinen Beitrag zu den politischen Bemühungen des letzten Dezenniums, an deren Ende der deutsch-polnische Vertrag und die kirchliche Neuregelung des Oder-Neiße-Gebiets standen. Auch die Mitglieder der Päpstlichen Kommission Justitia et Pax', wurden damit in die Lage versetzt, ihren Verpflichtungen gerecht zu werden, trotz bitterer Vergangenheit für den Völkerfrieden und die Eintracht unter den Katholiken zu arbeiten." ${ }_{1}$

Schauffs Tätigkeit für die ICMC und sein Engagement für die Sache Polens hatten eine konkrete gemeinsame Basis: Es ging auf beiden Ebenen um eine Lösung des Problems der sogenannten „Nationalgeschädigten“, zu denen auch die Personengruppe der nach 1945 in Deutschland befindlichen ehemaligen polnischen Zwangsarbeiter gehörte.

Um diesen Zusammenhang deutlich zu machen, ist ein kurzer Exkurs notwendig, zumal die Frage der „Zwangsarbeiter-Entschädigung“ ja bis heute virulent geblieben ist. Der Transfer polnischer Arbeitskräfte ins Reich und in die deutsche Wirtschaft während des Weltkriegs hatte schon bald die Form von Zwangsrekrurierung angenommen ${ }^{52}$. Insgesamt waren rund 1,6 Millionen polnische Zivilarbeiter in Deutschland eingesetzt. Hinzu kamen etwa eine halbe Million polnischer Kriegsgefangener, die ebenfalls zur Arbeit verpflichtet worden waren und rechtlich bald den gleichen Status erhielten.

Nach Kriegsende bildeten diese polnischen Arbeiter zusammen mit Fremdund Zwangsarbeitern aus anderen osteuropäischen Ländern (vor allem den „Ostarbeitern“ aus der Sowjetunion), soweit sie nicht zurückkehren konnten oder

50 Gregor XVI. (Bartolomeo Alberto Capellari), geb. 1765, Pontifikat von 1831 bis zu seinem Tode 1846.

51 Brief Schauff an Msgr. Giovanni Benelli, Sostituto nelle Segreteria di Stato, 14. November 1973 (Übers. aus dem Italienischen durch den Verfasser, IfZ, NL Schauff, Bd. 26).

52 Allgemein dazu Broszat, Polenpolitik, S. 102-117, und vor allem Herbert, Fremdarbeiter, S. $184 \mathrm{ff}$. 
wollten, das Gros jener in Deutschland gestrandeten Personengruppe, die von den Alliierten unter dem Begriff „Displaced Persons“ subsumiert und bis 1947 von der UNRRA und danach von der IRO betreut wurde. Die osteuropäischen und insbesondere die polnischen Zwangsarbeiter waren allerdings als sogenannte "Nationalgeschädigte“ bei den alsbald einsetzenden gesetzlichen Wiedergutmachungsbemühungen von deutscher Seite denjenigen gegenüber ins Hintertreffen geraten, die Entschädigungen für Verfolgungen aus politischen und rassischen Gründen einfordern konnten ${ }^{53}$ - in erster Linie die jüdischen Flüchtlinge, die bald auch besondere Privilegien in Hinblick auf Verpflegung und Rechtsstatus genos$\operatorname{sen}^{54}$.

Demarchen von polnischer Seite in dieser Frage, in denen die Auslegungspraxis deutscher Behörden beklagt wurde, Entschädigungen zu verweigern, weil Antragsteller nicht aus politischen, sondern aus „nationalen“ Gründen oder im Rahmen von allgemeinen sicherheitspolitischen deutschen Maßnahmen verfolgt worden seien, waren bereits früh erfolgt ${ }^{55}$. Sie blieben ohne direkten Erfolg. Die deutsche Seite vertrat völkerrechtlich die Position, daß Ansprüche nicht individuell geltend gemacht werden könnten, sondern nur durch den Staat, dem die Displaced Person angehörten. Im Falle Polens seien Reparationsforderungen staatlicherseits bereits durch die Sowjetunion abgefunden worden, zudem sei die Haftung der Bundesrepublik für im Ostblock wurzelnde Verbindlichkeiten angesichts der Bestimmungen des Potsdamer Abkommens völkerrechtlich fraglich ${ }^{56}$.

Das Erste Bundesentschädigungsgesetz von 1953 ermöglichte schließlich eine beschränkte Entschädigung auch von "Nationalgeschädigten“ bei dauerhaften Gesundheitsschäden. Bei der weiteren gesetzgeberischen Entwicklung, die 1956 in das Zweite Bundesentschädigungsgesetz mündete, war die Rolle der UNO sowie die Abstimmung mit den westlichen Alliierten, die Druck ausübten ${ }^{57}$, nicht ohne Bedeutung ${ }^{58}$. Die erreichten Verbesserungen waren allerdings marginal, ins-

$53 \mathrm{Vgl}$. Herbert, Nicht entschädigungsfähig?

54 Vgl. Jacobmeyer, Polnische Juden, S. 121 f.; allgemein zum Problem: Jacobmeyer, Vom Zwangsarbeiter zum heimatlosen Ausländer.

55 Vgl. das von dem Leiter der Rechtsabteilung der Londoner „Relief Society for Poles“, Wladislaw Borysiewicz, am 28. 3. 1950 dem Bundesjustizminister übermittelte „Memorandum über Wiedergutmachungsansprüche der polnischen Opfer des Naziterrors“, zit. in Jacobmeyer, Vom Zwangsarbeiter zum heimatlosen Ausländer, S. $232 \mathrm{f}$.

56 Diese Sicht der Dinge wurde durch ein Rechtsgutachten des Völkerrechtlers Ulrich Scheuner/Bonn untermauert: Eine Pflicht zur Wiedergutmachung bestehe nur von Staat zu Staat, wobei Polen durch das Potsdamer Abkommen gehindert sei, Ansprüche generell oder für Schäden von Privatpersonen geltend zu machen; durch die getroffenen alliierten Vereinbarungen seien auch der Bundesrepublik in dieser Angelegenheit die Hände gebunden (Jacobmeyer, Vom Zwangsarbeiter zum heimatlosen Ausländer, S. 233 f.).

57 So hatte der amerikanische Alliierte Hohe Kommissar am 10.12. 1953 nach Inkrafttreten des ersten Entschädigungsgesetzes moniert, daß die verfolgten Staatsangehörigen (allerdings der westlichen Länder) von Leistungen ausgeschlossen blieben (Herbert, Nicht entschädigungsfähig?, S. $284 \mathrm{f}$.).

58 Am 10. 9. 1951 hatte der Hohe Flüchtlingskommissar der UNO in Bonn eine Dépendance eröffnet und am 26. 5. 1952 wurde der „Vertrag zur Regelung der aus dem Krieg und der Besatzung entstandenen Fragen" zwischen den drei westalliierten Mächten und der Bun- 
besondere was die Polen betraf, denen weiterhin unter Berufung auf "national“ erkannte Verfolgung Entschädigungen verweigert wurden. Schließlich bemühten sich der UNO-Flüchtlingskommissar und die Bundesregierung gemeinsam um eine bessere Regelung der Frage der „Nationalgeschädigten“. Als Vertreter der ICMC wirkte Johannes Schauff an den Bemühungen um eine adäquate Lösung dieser Frage maßgeblich mit.

Am 5. Oktober 1960 schließlich wurde zwischen dem Hohen Kommissar der Vereinten Nationen für Flüchtlinge und der Bundesregierung ein Abkommen über Leistungen zugunsten von „Nationalgeschädigten“ geschlossen ${ }^{59}$. Die Entschädigungsberechtigung bei dauernden Schäden an Körper oder Gesundheit wurde gesetzlich anerkannt. Darüber hinaus stellte die Bundesregierung dem UNO-Flüchtlingskommissariat 45 Millionen Mark zur Verfügung, die der Gruppe der „Nationalgeschädigten“ und ihren Angehörigen zugute kommen sollten, sofern sie Flüchtlinge im Sinne der Genfer Konvention vom 28. Juli 1951 seien. Der neue Anspruch der "Nationalgeschädigten“ und die finanzielle Hilfestellung waren allerdings nicht aufgrund eines Gesetzes zustandegekommen, sondern aufgrund eines Vertrages. Der Versuch einer endgültigen gesetzlichen Regelung wurde mit dem Dritten Bundesentschädigungsgesetz bzw. dem Bundesentschädigungs-Schlußgesetz von 1965 unternommen: Das zentrale Anliegen des Gesetzes von 1960 hinsichtlich Wiedergutmachung bei Schädigung der Gesundheit wurde noch einmal bestätigt und darüber hinaus der Anspruch auf rückständige Rentenbezüge anerkannt; Antragstellungen sollten bis zum 30. September 1966 erfolgen ${ }^{60}$.

Die Durchführung dieses Schlußgesetzes erwies sich allerdings als problematisch. Dies betraf vor allem die für die Betroffenen komplizierte und schwierige Beweisführung des Tatbestandes der Verfolgung und der Schädigung, da 80 Prozent der Antragsteller außerhalb der Bundesrepublik im „Ostblock“ lebten. Ebenso schwer wog, daß die deutsche Seite weiterhin an dem Grundsatz festhielt, Zwangsarbeit aus „nationalen“ Gründen könne kein Entschädigungsgrund sein. Die Frage des Lohnausfalls bzw. der Diskriminierung bei Lohnauszahlungen wurde von deutscher Seite dem Reparationskomplex zugeordnet, der in dem Londoner Schuldenabkommen vorläufig zurückgestellt worden war ${ }^{61}$.

Im Zentrum der deutschen Wiedergutmachungspolitik standen die Juden. Bis in die siebziger Jahre wurde nur etwa ein Drittel der Anträge von „Nationalgeschädigten" positiv beschieden ${ }^{62}$. Doch wäre auch dieser nur bescheidene Erfolg vielleicht nicht möglich gewesen ohne das Engagement einiger bundesdeutscher

desrepublik Deutschland (Überleitungsvertrag) unterschrieben. BGBl II 1954, S. 7 f. und $194 \mathrm{f}$.

59 Bulletin des Presse- und Informationsamtes der Bundesregierung, Nr. 190, S. 1837, in: Schirilla, Wiedergutmachung, S. 54-57.

60 BGBl I 1965, S. 1315; Schirilla, Wiedergutmachung, S. $67 \mathrm{ff}$.

61 Im Londoner Schuldenabkommen vom 27. 2. 1953 war die Priorität der Reparationsfrage vor allen anderen Forderungen festgelegt worden. Zu den Konsequenzen für Wiedergutmachungsansprüche vgl. Herbert, Nicht entschädigungsfähig?, S. $278 \mathrm{ff}$.

62 Aufschlüsselung der Zahlen bei Schirilla, Wiedergutmachung, S. 76. 
Politiker und Kirchenführer, an dem Johannes Schauff aufgrund seiner in der Flüchtlingsarbeit gewonnenen Erfahrungen wesentlich beteiligt war.

Eine von Schauffs vorrangigen Aufgaben in der ICMC war die Organisierung der Weiterwanderung in Deutschland verbliebener DPs vor allem in die Neue Welt. Wie bereits erwähnt, arbeitete er dabei eng mit dem die Auslandspolen betreuenden Bischof Josef Gawlina zusammen. Daß viele Mitarbeiter der ICMC Polen waren, hat ebenfalls mit Schauffs wachsender Sensibilität gegenüber diesem spezifischen Problem zu tun. Verbündete für die polnische Sache fand er jedoch vor allem in Deutschland selbst. Hier hatte er Zugang zu Kardinal Frings und dem Kommissariat der Deutschen Bischöfe unter der damaligen Leitung des Münsteraner Weihbischofs Heinrich Tenhumberg. Rechtsanwalt Herbert J. Becher als Vertreter des Katholischen Büros des Kommissariats war von Schauff bereits sehr früh für diese Problematik sensibilisiert worden und wurde zu einem der engagiertesten Verfechter der polnischen Sache ${ }^{63}$. Im Bundestag setzten sich einige Abgeordnete für die Wiedergutmachung an den "Nationalgeschädigten“ ein, darunter wiederum Herbert Czaja ${ }^{64}$.

Schauff vermochte aber auch im internationalen Rahmen Kräfte zu mobilisieren. Dazu gehörten die Verbindungen der Bundesrepublik zur UNO - etwa im Falle der Vereinbarung mit dem Hohen Flüchtlingskommissar vom Oktober 1960, zu der wiederum Schauffs gute Beziehung zu Außenminister Brentano beigetragen hatte. Aber auch Schauffs Verbindungen nach Amerika und dort vor allem zu Kreisen der konservativen deutschen Emigration, die sich in der Frage der polnischen "Nationalgeschädigten " nutzen ließen, spielten eine nicht unerhebliche Rolle65. Dennoch: Von den bis 1986 gestellten 36. 812 Anträgen auf Entschädigung für "Nationalgeschädigte“ wurden 83 Prozent abgelehnt ${ }^{66}$. Viele der Betroffenen waren verbittert, und der Konflikt ist bis heute noch nicht endgültig gelöst ${ }^{67}$.

Schauffs Engagement zum Abbau der historisch belasteten deutsch-polnischen Gegensätze führte dennoch zu beachtlichen Ergebnissen. Mit der politischen Entwicklung in Polen nach dem Aufbruch von Solidarnosc lockerte sich schließlich zunehmend die unselige Verbindung von Kirche und Regime. Das Scheitern der Bewegung löste in Deutschland eine Welle von Sympathie und Hilfsbereitschaft aus, bei der auch deutscherseits Ressentiments zurücktraten.

Nach dem Tode der zentralen Dialogpartner auf polnischer Seite, Kominek und

63 IfZ, NL Schauff, Bd. 32.

64 Vgl. u.a. Verhandlungen des Deutschen Bundestags, 5. Wahlperiode, Stenographische Berichte, Bd. 70, 246. Sitzung, 2. Juli 1969, S. 13716-13719.

$65 \mathrm{Vgl}$. u. a. die Intervention des Abgeordneten Pucinski/Illinois im US-Repräsentantenhaus vom 17. 10. 1969, die sich auf ein Memorandum des Katholischen Büros zur Entschädigung der Nationalverfolgten bezieht. Abgedruckt in Schirilla, Wiedergutmachung, S. 130139; Becher, Freund Polens, S. 243 f.; Korrespondenz Schauff-Becher sowie SchauffCzaja, IfZ, NL Schauff, Bd. 1 und 26.

66 Herbert, Nicht entschädigungsfähig?, S. $301 \mathrm{f}$.

67 Vgl. dazu u. a. die Artikel in der Süddeutschen Zeitung vom 21./22. 7. 1996, 6. und 7. 11. 1997 sowie die Debatten um das Gesetz zur Errichtung der Bundesstiftung „Erinnerung, Verantwortung, Zukunft". 
Wyszynski ${ }^{68}$, sieht der Journalist Hansjakob Stehle jedoch, offenbar zu Recht, daß angesichts einer relativen Normalisierung der zwischenkirchlichen Beziehungen die Mohren ihre Schuldigkeit getan hatten. Als Deutsche und Polen 1982 in Rom gemeinsam die Heiligsprechung des in Auschwitz ermordeten Franziskanermönchs Maximilian Kolbe feierten, habe der Krakauer Kardinal Macharski zwar an die verstorbenen Wegbereiter der deutsch-polnischen Annäherung erinnert, zu denen er auch Kardinal Döpfner zählte. Zu dem Empfang, den die deutschen Bischöfe ihren polnischen Amtsbrüdern aus diesem Anlaß gaben, sei neben anderen engagierten deutschen Laien - Johannes Schauff jedoch nicht eingeladen worden, der sich für die deutsch-polnischen Kontakte „wie kaum ein anderer" eingesetzt habe ${ }^{69}$.

68 Boleslaw Kominek starb am 10. 3. 1974, Kardinal Wyszynski am 28. 5. 1981.

69 Stehle, Johannes Schauff, S. 243. 